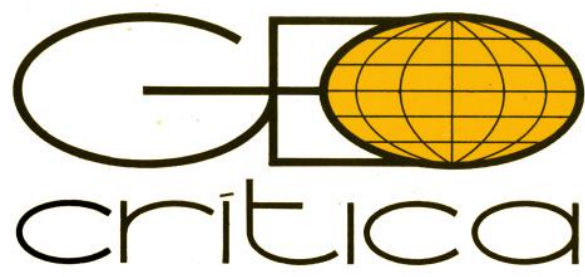

\title{
LAS MULTINACIONALES Y LAS RELACIONES CON SUS FILIALES
}

\author{
Ángel Calvo \\ Universitat de Barcelona \\ angel.calvo@ub.edu
}

\section{Las multinacionales y las relaciones con sus filiales (Resumen)}

Las multinacionales son empresas que desempeñan una parte variable de su actividad fuera de las fronteras de los países en los que tienen sus sedes centrales. Están formadas por filiales, subsidiarias o asociadas dispersas por el globo terráqueo. El objetivo de este artículo es recoger los ejes centrales del debate en torno a las relaciones existentes entre la casa madre y el segundo grupo. En este sentido, se pone énfasis en la cuestión de la transferencia de conocimiento e innovación. El texto se estructura en dos grandes apartados, que contienen una panorámica general y un examen de las relaciones entre las multinacionales y las empresas locales o nacionales.

Palabras clave: Multinacionales; filiales; transferencia de conocimiento; transferencia inversa.

\section{Multinationals and relations with their subsidiaries (Abstract)}

Multinationals are companies that carry out a variable part of their activity outside the borders of the countries where they have their headquarters. They are made up of subsidiaries, affiliates or associates scattered around the globe. The aim of this article is to set out the central themes of the debate on relations between the parent company and the second group of units. In this sense, emphasis is placed on the issue of knowledge transfer and innovation. The text is structured in two main sections, containing a general overview and an examination of the relations between multinationals and local or national companies.

Keywords: multinationals; subsidiaries; knowledge transfer; reverse transfer. 


\section{Introducción}

La Real Academia Española define las multinacionales como sociedades mercantiles o industriales con intereses y actividades establecidos en numerosos países. Esta definición se basa en el aspecto geográfico y no contempla precisiones sobre la índole de los intereses y actividades. Más impreciso, el término transnacional señala que se extiende a través de varias naciones.

Por su parte, el Cambridge Dictionary califica como "multinational" "una empresa muy grande que tiene oficinas, tiendas, etc. en muchos países diferentes pero que es controlada desde el país donde se inició" e incluso llega a precisar el tipo y volumen de actividad implicado (una "empresa con un cuarto o más de sus ventas en otros países").

Si miramos en Internet, la voz "multinacionales" arroja aproximadamente 7.190.000 resultados en 0,49 segundos y en inglés aproximadamente 82 millones de resultados en 0,73 segundos. En un buscador académico más específico -Google Scholar- los resultados obtenidos se elevan a 313.000 en 0,05 segundos. La búsqueda 'multinationals' acotada con 'local subsidiaries' da aproximadamente 148.000 resultados en 0,08 segundos. La expresión "local subsidiaries of multinational corporations, autonomy" da unos 59.100 resultados en 0,11 segundos. Aquí irrumpen con vigor algunos temas centrales, como el de la I+D y las economías emergentes de Asia. Siendo impresionantes, estos resultados resultan discretos. La naturaleza interactiva de la red lleva a incrementar los resultados a medida que las consultas se multiplican. Días después de la primera búsqueda y desde el mismo dispositivo, el término 'multinationals' acotado con 'local subsidiaries' arroja aproximadamente 12,8 millones de resultados $(0,73$ segundos $)$.

Sin necesidad de acotar el término, en la primera decena de títulos ya aparecen algunas connotaciones importantes de estas empresas. Destacan la relación con el crecimiento económico en general y con el país de acogida, en particular ${ }^{1}$, la irrupción de los mercados emergentes como destino de la inversión directa $^{2}$ y como nuevos protagonistas de la economía, las finanzas, el comercio y las inversiones mundiales -los dragones asiáticos y las multilatinas- ${ }^{3}$, por no olvidar la elección del modo de acceso a los mercados correspondientes $^{4}$. Recogen, asimismo, algunas realidades importantes, como las multinacionales regionales 5 , y cuestionan argumentos muy arraigados, como el de la identificación de multinacionales y ventajas específicas ${ }^{6}$.

En otras palabras, aunque de ellas solo una pequeña parte es accesible, resulta imposible intentar determinar el alcance y contenido del tipo y actividad de estas empresas, así como de la miríada de implicaciones que entrañan. Por lo tanto, acotar bien la tarea parece el procedimiento natural para lograr un buen resultado.

Así, pues, nos proponemos dar cuenta de los resultados que contiene la red sobre las multinacionales desde la óptica de historia de la empresa. Dentro de esta perspectiva, nos ocupamos de las relaciones entre las sedes centrales de las multinacionales y las filiales o

\footnotetext{
${ }^{1}$ Rodriguez-Clare 1996, p. 852-873.

${ }^{2}$ Ramamurti y Singh 2009.

${ }^{3}$ Mathews 2006.

${ }^{4}$ Kim, Hwang 1992, p. 29-53; subrayamos el fenómeno de las multilatinas: Andonova y Losada-Otálora 2017.

${ }^{5}$ Rugman 2005.

${ }^{6}$ Fosfuri, Motta 1999, p. 617-630.
} 
"geographically dispersed value-adding subunits". Se pone énfasis en la cuestión de la transferencia de conocimiento e innovación ${ }^{7}$. El texto se estructura en dos grandes apartados, que contienen una panorámica general y un examen de las relaciones entre las multinacionales y las empresas locales.

Se trata, ni más ni menos, que de ampliar y sistematizar un esfuerzo que da mayor solidez a la investigación empírica sobre el tema. Tomemos como ejemplo la investigación propia sobre las Tecnologías de la Información y la Comunicación en España -eje de la economía, la sociedad y la vida cotidiana de las personas- en los dos decenios finales del siglo veinte y los años iniciales del nuevo milenio. Se trata de una serie de trabajos realizados desde una perspectiva interdisciplinar y una metodología de estudio de caso. Destaca, asimismo, la variedad de fuentes utilizadas, que incluyen las primarias de procedencia diversa -las propias empresas, la administración o las instituciones-, la fuente oral, la hemeroteca y la bibliografía secundaria. Con idéntico planteamiento y metodología, dos nuevos trabajos aceptados en revistas internacionales permitirán engrosar la lista con otros tantos nombres de empresas, a saber, Telettra Española y Fujitsu Española, filiales de las multinacionales Telettra SpA y Fujitsu Ltd., con sede en Italia y Japón, respectivamente ${ }^{8}$.

El conjunto de estudios brinda las vicisitudes de un nutrido grupo de empresas, la mayoría en calidad de protagonistas de subsectores distintos. Un denominador común al conjunto estriba en el fenómeno de las multinacionales, sea en su calidad de matrices de las filiales nacionales, sea en su naturaleza de empresas con sede en el país anfitrión. En un segundo aspecto, las empresas estudiadas comparten su preocupación por actuar de puntas de lanza contra la dependencia tecnológica, objetivo que en raras ocasiones se cumple. En la bibliografía se recogen los textos publicados con sus correspondientes enlaces.

\section{Panorámica general}

Como es natural, el arranque de la construcción de un marco general apela a una breve incursión en la plasmación histórica del fenómeno de las multinacionales. En los decenios de 1960 y 1970 las multinacionales lograron erigirse en protagonistas en el escenario mundial hasta el punto que el valor total de la producción internacional controlada por esas empresas llegó a rebasar el del comercio internacional. Su difusión y crecimiento ha sido uno de los fenómenos sobresalientes de los últimos tiempos ${ }^{9}$.

Esta posición central en el mundo actual ha llevado a los especialistas a hacer hincapié en las relaciones entre estos gigantes y las empresas locales en la vertiente de control directo desde

\footnotetext{
${ }^{7}$ Una visión general en Kostova et al. 2016. La corriente de investigación centrada en las filiales (véase una revisión en Paterson y Brock 2002) estuvo encabezada por la investigación sobre la internacionalización de las actividades innovadoras y otros tipos de unidades influyentes (Birkinshaw y Hood 1998; Frost 1998; Holm y Pedersen 2000); algunos (Hansen y Nohria 2004, p. 95) consideran a las filiales como "partes de una cadena de valor añadido mundial". El debate se ha prolongado en el tiempo: Dzedek 2018. Resulta de interés subrayar que el debate sobre las filiales contribuyó a modular las concepciones contemporáneas de la empresa multinacional (Hedlund 1986; Bartlett y Ghoshal 1989; Doz et al. 2001). La internacionalización afianza las ventajas de la multinacional proporcionando oportunidades para dividir los riesgos del mercado al trocear la cadena de valor y facilitar el acceso a nuevos recursos y activos: Rugraff y Hansen (eds.), 2011, p. 21-24.

${ }^{8}$ Calvo 2009, p. 43-72; 2019, p. 29-47; 2019a, p. 130-152; 2019b, p. 142-179; 2020, p. 81-94; 2020a, p. 1-13; 2020b, p. 1-25; 2020c, p. 1-26; 2020d, p. 1-20 y 2020e, p. 219-262.

${ }^{9}$ La doctrina oficial de las Naciones Unidas (United Nations 1974, p. 5) afirmaba el papel ambivalente de las empresas multinacionales, como instrumentos eficaces de desarrollo y fuentes de tensión o conflicto.
} 
la sede o de control indirecto mediante la expatriación. En general, los estudiosos consideran las multinacionales como empresas con establecimientos y activos fuera de la sede y las filiales, en tanto que activos de las multinacionales, como empresas más pequeñas bajo la autoridad y la gestión de la matriz ${ }^{10}$. Pero simplemente la consideración del tamaño y grado de control de la matriz ya pueda dar lugar a controversias.

Parece natural, en primer lugar, recurrir a instrumentos que sirvan de guía a la indagación sobre las diversas problemáticas. En este sentido, destacan las enciclopedias y, entre ellas The Palgrave Encyclopedia of Strategic Management, a la que recurriremos más adelante.

Parece, igualmente, natural orientarse a panorámicas generales que comprendan periodos amplios de tiempo. En este sentido, el estudio de las relaciones de las sedes desde las páginas del Journal of World Business (JWB), conocido como el Columbia Journal of World Business (CJWB), hasta 1997 muestra una evolución desde los mecanismos más burocráticos y formales hasta los más interconectados e informales. Ante el creciente y cada vez más globalizado mercado internacional, las empresas multinacionales han aprovechado las mejoras en la tecnología de la información y las comunicaciones, reduciendo las barreras al comercio y la inversión. Asimismo, han aumentado la experiencia organizativa en las operaciones internacionales y los directivos y empleados cada vez más formados y experimentados para aflojar los controles internos formales en nombre de una mayor eficiencia organizativa, motivación e innovación. Los principales temas y tendencias de la investigación sobre las relaciones entre la sede central y el personal directivo publicado en CJWB/JWB a lo largo del tiempo muestra cinco temas dominantes: diseño organizativo y sistemas de control; contexto del país de origen y del país anfitrión; funciones subsidiarias y estructuras regionales; creación y transferencia de conocimientos o transferencia inversa y gestión de expatriados y gestión global de los recursos humanos ${ }^{11}$.

A lo largo del medio siglo objeto de examen, se pasó de una conceptualización de la relación entre las filiales y la sede central de las empresas multinacionales que se basaba en las ideas del paradigma de "estrategia-estructura"12 y en los sistemas formales de control y coordinación de las filiales extranjeras a un mayor interés y apreciación de las complejidades asociadas con la gestión de los entornos internos y externos de las empresas multinacionales que surgieron en trabajos posteriores. Se puso mayor hincapié en modelos de gestión más colaborativos - modelos metanacionales ${ }^{13}$, transnacionales ${ }^{14}$ y heterárquicos ${ }^{15}$-, cimentados en

\footnotetext{
${ }^{10}$ Khan 1986, p. 15-25.

11 Kostova et al. 2016. Por transferencias inversas de conocimientos nos referimos a intercambios intrainstitucionales de información, conocimientos técnicos o tecnología de filiales internacionales (países anfitriones) a las sedes de las empresas (países de origen): Ambos 2018. El término 'transferencia inversa' ('reverse transfer') fue acuñado por Hakanson y Nobel 2001 y Yamin 1995.

${ }^{12}$ La estructura -esquema organizativo para la gestión eficiente de una empresa- sigue a la estrategia -objetivo de una empresa a largo plazo-: Chandler 1962.

${ }^{13}$ Doz, Santos y Williamson 2001. Las empresas metacionales no sacan su ventaja competitiva de su país de origen, ni siquiera de un conjunto de filiales nacionales. Se mueven en un escenario global, salpicado de bolsas de tecnología, inteligencia de mercado y capacidades, un potencial por explotar: Birkinshaw et al. (ed.) 2004, p. 155.

${ }^{14}$ En el aspecto organizativo, una empresa transnacional es una categoría de multinacional compuesta por una red integrada e interdependiente de filiales en el mundo entero, que ejercen funciones estratégicas y actúan como centros de excelencia mediante el intercambio eficaz de conocimientos y experiencia entre ellas filiales. Unilever ofrece un buen ejemplo de empresa transnacional: Bartlett y Ghoshal 1989. Las empresas multidomésticas, ejemplificadas por Nestlé, se distinguen por una estructura muy descentralizada y poco vinculada, en la que las filiales de todo el mundo funcionan de manera relativamente autónoma e independiente de la sede central.
} 
la idea de que las filiales desempeñan un papel estratégico muy importante debido al acceso de las filiales a recursos únicos. Esta visión siguió fortaleciéndose a partir del decenio de 1980, al subrayar las funciones especializadas de las filiales extranjeras y sus contribuciones singulares al éxito general de la empresa ${ }^{16}$.

Especial atención ha merecido el estudio de los microfundamentos de los procesos de creación y coordinación de conocimientos en las empresas multinacionales ${ }^{17}$. Esfuerzos no desdeñables se han orientado a examinar las consecuencias de la transferencia de conocimientos para las filiales extranjeras ${ }^{18}$.

En el segundo decenio del siglo XXI, apareció un novedoso enfoque del estudio de los procesos de micronivel en las organizaciones. La exploración del lenguaje como un tema independiente y no solo como un componente de la distancia cultural y su papel en los esfuerzos de transferencia de conocimientos en las $\mathrm{EMN}^{19}$ permitió aclarar aspectos de los desafíos de la creación y difusión de conocimientos ${ }^{20}$. Se constató así la índole multilingüe de las empresas multinacionales a la vez que la variación considerable de la fluidez de los idiomas según las funciones y los niveles de organización ${ }^{21}$.

\section{Multinacionales y empresas locales}

La investigación sobre las relaciones entre las empresas multinacionales y las empresas locales -entendidas como asentadas en el país anfitrión- se basa principalmente en tres tradiciones económicas, a saber, el comercio internacional ${ }^{22}$, la organización industrial (la obra fundamental de Stephen Hymer en 1960 hasta el marco "ecléctico" de la OLI de Dunning, incluidos Buckley y Casson, Kogut y Zander, y la economía del comercio, que más tarde incluyó los movimientos de capital. A partir de Hymer, la incipiente bibliografía de International Business atribuía a las multinacionales activos especiales en comparación con las empresas locales que les permiten superar la desventaja de ser extranjeras ${ }^{23}$. En los últimos años, estas tres tradiciones han inspirado una literatura teóricamente empírica centrada en el nexo entre las empresas multinacionales y locales mediante tres métodos principales: la modelización formal, el análisis estadístico y los estudios de caso como metodología. Los tres métodos se cimientan en razonamientos de diferentes tipos sobre

\footnotetext{
${ }^{15}$ En las empresas heterárquicas el poder y los privilegios se distribuyen a muchos puntos de una red, en lugar de asignarlos a los miembros situados en lo alto de la estructura. Predomina la coordinación horizontal frente a la lógica de arriba hacia abajo o de abajo hacia arriba: Hedlund 1986.

${ }^{16}$ Birkinshaw 2009, p. 367-388.

${ }^{17}$ Johnson y Duxbury 2010; Klitmøller y Lauring 2013; Tippmann et al. 2014; Zander et al. 2012.

${ }^{18}$ Chen et al. 2012; Ciabuschi et al. 2012; Fang et al. 2013; Najafi-Tavani et al. 2014.

19 Harzing y Pudelko 2013.

${ }^{20}$ Barner-Rasmussen y Aarnio 2011.

21 Harzing et al. 2011, Heikkilä y Smale 2011, Yamao y Sekiguchi 2015.

${ }^{22}$ Caves 1971, p. 1; Bain 1956. Bain fue duramente criticado desde las filas de las Universidades de Chicago y Harvard. Los primeros trataron de poner en pie nuevas propuestas teóricas mientras que los segundos pugnaron por demostrar la existencia de barreras de entrada: Rosado (2015). Ghoshal y Bartlett (1988, p. 1 y 21) sugieren cuatro atributos de una filial nacional, a saber: el alcance de los recursos locales escasos, la autonomía local en la adopción de decisiones, la integración normativa de la filial con los objetivos y valores de la empresa matriz y la densidad de la comunicación interna entre los directivos de la filial y la densidad de su comunicación con los directivos de la sede central y otras filiales de la empresa.

${ }^{23}$ Hymer 1960; Dunning 1988; 2001; Buckley y Casson 1976; Kogut y Zander 1993, MacDougall 1960; Aliber 1971.
} 
cuestiones fundamentales, entre ellos el uso de la lógica inductiva y deductiva, la selección de casos y la conceptualización de las variables ${ }^{24}$.

En particular, Rodriguez-Clare (1996) formaliza el concepto de encadenamientos hacia atrás y hacia adelante. El primero significa que en tres circunstancias relativas a los inputs -variedad en la producción de bienes finales; compra por parte de las empresas nacionales de todos ellos a nivel local y producción de los mismos con rendimientos crecientes a escala-, al aumentar la demanda de inputs, una empresa de bienes finales genera una externalidad positiva a otros productores de bienes finales. Por eslabonamientos hacia adelante se entiende que la producción local de inputs más especializados permite la producción de bienes que utilizan con alta intensidad inputs especializados a costes competitivos ${ }^{25}$.

El análisis estadístico confirmó varias conclusiones de estudios anteriores realizados con datos más agregados e identificó nuevos factores determinantes de la propensión a internalizar las transacciones en las empresas internacionales. Se ha puesto en evidencia que la compra de las filiales a sus empresas matrices aumenta con los gastos de estas en I+D. Por otra parte, el grado de multinacionalidad de las empresas matrices afecta negativamente a esas importaciones. Finalmente, se carece de pruebas estadísticas para rechazar la hipótesis de que no existen diferencias significativas entre los países en desarrollo y los países menos adelantados ${ }^{26}$.

Al inicio del ascenso de las multinacionales, los investigadores de la corriente dominante consideraban que las ventajas específicas de la propiedad se desarrollaban en la empresa madre y se explotaban en el extranjero mediante la transferencia de tecnología y conocimientos "hacia adelante", es decir de la sede central a las filiales en el extranjero ${ }^{27}$. Los países en desarrollo sostienen que la transferencia de tecnología por las multinacionales no les supone ningún coste adicional significativo porque esa tecnología ya se ha producido. En una visión opuesta, las empresas multinacionales tratan de obtener el mejor precio posible por su tecnología. Las grandes instituciones internacionales recordaban que la transferencia de tecnología tiene lugar en un mercado muy imperfecto en el que los países en desarrollo presentan una posición negociadora débil frente a las empresas multinacionales ${ }^{28}$.

Con el tiempo, algunos se percataron del papel fundamental que desempeñaban muchas filiales en la competitividad de sus empresas, y la investigación académica comenzó a ponerlo de relieve desde un enfoque evolutivo (Bartlett y Ghoshal, 1989). Se habla de la transferencia inversa de conocimientos, es decir, desde las filiales extranjeras a las empresas matrices, un mecanismo por el cual las filiales pueden mejorar su estatus y reclamar recursos, a la vez que contribuir a fortalecer su posición competitiva de la empresa en su conjunto ${ }^{29}$.

\footnotetext{
${ }^{24}$ Para los estudios de caso, véase Altenburg, 2000; Hansen and Schaumburg-Müller, 2006); para visiones generales, véase Blomström y Kokko, 1997.

${ }^{25}$ Rodriguez-Clare 1996, p. 853.

${ }^{26}$ Blomström y Kokko 2000, p. 77; Rugraff y Hansen, eds. 2011.

${ }^{27}$ Vernon 1966, p. 190-192; Dunning 1981, p. 1-22. Más tarde, en 1971, Raymond Vernon formuló el modelo de negociación obsolescente (OBM), que explica la naturaleza cambiante de las relaciones de negociación entre una empresa multinacional y el gobierno del país anfitrión en función de los recursos, los objetivos y las limitaciones de ambas partes. La transferencia de tecnología es una condición del crecimiento económico de cada país: Teece 1977, p. 242.

${ }^{28}$ United Nations 1973.

${ }^{29}$ Edwards y Tempel 2010; Ghoshal y Bartlett 1988a, p. 365-388. Las filiales nacionales intervienen en la creación de nuevos productos, procesos o sistemas administrativos; la adopción de innovaciones de la empresa
} 
$\mathrm{Al}$ análisis de la transferencia inversa se ha dedicado atención en los últimos años ${ }^{30}$ y a ello ha contribuido la investigación sobre las multinacionales de mercados emergentes - consideradas por numerosos investigadores como los nuevos motores ocultos del comercio mundial y el crecimiento económico ${ }^{31}$.

La creciente presencia de los países en el escenario mundial y el advenimiento de las llamadas economías en transición, principalmente con la caída del Muro de Berlín, contribuyeron a reavivar el debate ${ }^{32}$. Desde finales del decenio de 1990, la investigación ha esclarecido en gran medida el papel que desempeñan las filiales dispersas internacionalmente en la acumulación de conocimientos de las sedes centrales ${ }^{33}$. Las filiales aparecen como canal de acceso al conocimiento y a la tecnología situada en los mercados locales de las filiales ${ }^{34}$.

Diversos estudios se han centrado en los antecedentes, la cantidad y los factores de éxito de las transferencias inversas de conocimientos considerando el país anfitrión como determinante para la obtención y creación de conocimientos y las funciones de la filial. Se ha comprobado que los mecanismos de coordinación (Ambos et al., 2006; Asmussen et al., 2013) y la capacidad de absorción de la sede central desempeñan un papel crucial. El estudio de las variables relacionales, llámese distancia geográfica o cultural entre la sede y las filiales, ha llevado al reconocimiento de la importancia de las transferencias inversas en general y a la conciencia de que la dirección de la transferencia es una variable clave en el estudio de los flujos transfronterizos de conocimiento en la empresa multinacional.

Más recientemente, los especialistas examinan la forma en que las filiales de las empresas multinacionales experimentan interacciones con las sedes de las empresas. Constatan que el clima de relación reduce significativamente los costes de organización - negociación e información-, que la distancia aumenta los costes de negociación y que la centralización y la formalización reducen los costes de información, mientras que la integración social aumenta los costes de negociación ${ }^{35}$. En último lugar, algunos estudios atribuyen un impacto positivo sobre el crecimiento de la productividad tanto a la I+D de la filial como a la transferencia de tecnología dentro de la empresa matriz, a la vez que corroboran la complementariedad de ambas fuentes de tecnología ${ }^{36}$.

Un aspecto importante del debate internacional se refiere a los mecanismos de control utilizados por las multinacionales. A finales del decenio de 1990, la mayoría de los estudiosos destacaron la estructura y la estrategia de las empresas multinacionales, el papel de los

matriz y la difusión de las innovaciones de las filiales: Ghoshal y Bartlett 1988, p. 1 y 21. De la misma manera que se habla del papel de los expatriados de las mutinacionales, se puede hablar de la función de los impatriados: Reiche 2011, p. 365-389.

${ }^{30}$ A partir del caso español, Jimenez, Martínez-Costa y Sanz (2019, p. 629-648) encuentran una relación positiva entre la transferencia inversa de conocimientos y la innovación de la matriz. Esta relación se acrecienta con la naturaleza tácita de los conocimientos transferidos y con la distancia organizativa entre ellas.

${ }^{31}$ Freitas et al. 2017, p. 176-188; Boston Consulting Group 2012; Bonaglia et al. 2007; aproximación desde la India: Nair y Mellahi 2015.

${ }^{32}$ Rugraff y Hansen, eds. 2011.

${ }^{33}$ Ambos 2018; una revisión de la bibliografía, véase Kogut y Cotta de Mello 2017, p. 1.590-1.807.

${ }^{34}$ Borini et al. 2012; Criscuolo y Narula 2007; Frost y Zhou 2005.

${ }^{35}$ Lunnan et al. 2019, p. 1-24.

36 Belderbos et al. 2008, p. 310-319. De esta forma, el uso de una fuente de tecnología aumenta el impacto marginal de la otra. 
expatriados, la transferencia internacional de gerentes y la gestión internacional de los recursos humanos ${ }^{37}$.

Diversos estudios realizados desde el comienzo del nuevo milenio muestran que las sedes de las empresas multinacionales tratan de ejercer cierto grado de control sobre las filiales extranjeras ${ }^{38}$. Todo ello suele estar determinado, en diversos grados, por una mezcla de características que abarcan aspectos geográficos (país de origen y país anfitrión) y sectoriales (factores específicos de la industria y la empresa). Además, las investigaciones muestran que las empresas multinacionales de los distintos países tienen diferentes pautas, grados y tipos de control sobre sus filiales extranjeras ${ }^{39}$.

Aportaciones al debate descubren similitudes y diferencias en la gestión de las empresas multinacionales. Tanto las multinacionales estadounidenses como las europeas muestran un alto grado de descentralización; sin embargo, en el caso de las empresas europeas el grado de descentralización es mayor que en las empresas multinacionales estadounidenses ${ }^{40}$. Con todo, las empresas siguen trayectorias diversificadas. Inicialmente, Hewlett-Packard Company encomendó a expatriados estadounidenses la gestión de sus filiales extranjeras, a la vez que apostó por gestores nacionales locales y por sustituir gradualmente a los expatriados. Esta práctica se reveló ventajosa por varias razones: aprovecha los conocimientos y contactos locales, aporta mayor estabilidad y continuidad y es menos costosa ${ }^{41}$. Emplear gerentes locales fue una práctica permanente de IBM en Brasil, al lado de otros principios básicos para hacer negocios en todo el mundo como la ambición y la anticipación ${ }^{42}$.

Tradicionalmente, se ha hecho hincapié con bastante frecuencia en las especificidades del Japón, en comparación con las de los Estados Unidos y Europa, en las prácticas de gestión. Una investigación en gran escala sobre la organización y el control de las filiales en China por parte de las empresas multinacionales japonesas y europeas tan sólo revela un corto número de diferencias significativas, relativas principalmente a la capacitación del personal local y a las políticas de expatriación ${ }^{43}$. Si consideramos las filiales japonesas en Estados Unidos, numerosos expatriados encuentran dificultades en el trato con la mano de obra del país anfitrión, lo que les obliga a anticipar el regreso al país de origen. Asimismo, la relación entre gerentes japoneses expatriados y estadounidenses locales se ve salpicada de conflictos en algunas de las filiales japonesas en Norteamérica ${ }^{44}$.

\footnotetext{
${ }^{37}$ Harzing 1999.

${ }^{38}$ Child, Failkner y Pitkethly 2000; Farley, Hoenig y Yang 2004; Gamble 2003; O'Donnell 2000 y O’Donnell y Blumentritt 1999, p. 187-206.

${ }^{39}$ Egelhoff 1984; Vachani 1995; Harrison y McKinnon 1999. Egelhoff señala dos tipos de control comúnmente utilizados en las multinacionales: los sistemas de información sobre el rendimiento y la asignación de los directivos de la empresa matriz a las filiales extranjeras.

${ }^{40}$ Shetty 1979, p. 39-48. Para tomar un ejemplo, General Electric de la creación de mini empresas de respuesta local y autosuficientes en cada mercado, la empresa pasó a desarrollar suministros de bajo coste en el extranjero. Sin embargo, en Brasil, el empeño de los gerentes locales para responder a los cambios de las circunstancias se vio anulado por otras prioridades de la sede: Stopford, Strange y Henley 1991, p. 141.

${ }^{41}$ Rosenzweig 1994, p. 107-123. Unilever se organizaba de forma descentralizada. Las empresas filiales en los principales mercados nacionales se encargaban de la producción, la comercialización, la venta y la distribución de los productos en ese mercado. Para impulsar la localización, Unilever contrató a gerentes locales para dirigir las filiales en el mundo: Hill 2014, p. 465.

${ }^{42}$ https://www.ibm.com/ibm/history/ibm100/us/en/icons/ibmworldtrade/

${ }^{43}$ Jaussaud y Schaaper 2007, p. 223-245.

${ }^{44}$ Paik y Sohn 2004.
} 
A lo largo de los años, las investigaciones han aumentado considerablemente, pero los resultados han seguido siendo fragmentarios $\mathrm{y}$, en cierta medida, contradictorios. La bibliografía comprende el análisis de cuestiones específicas, como las relaciones entre la sede central y las filiales ${ }^{45}$, o regiones determinadas ${ }^{46}$. Un campo de investigación diferente compara la administración de la gestión entre regiones ${ }^{47}$.

La situación económica de un país conforma el control de la gestión. Tras la crisis económica de 2009, las multinacionales occidentales centralizaron el control de la gestión y los expatriados en las filiales extranjeras, que fueron sustituidos en gran medida por gerentes locales y formación adicional (Schaaper et al., 2011).

Por último, un examen sistemático de la bibliografía permite a Sageder y FeldbauerDurstmüller (2019, p. 875-918) vincular el planteamiento del control de la gestión y la responsabilidad de esta a factores internos de la sede y la filial, así como de factores externos como la cultura o las exigencias del mercado. La relación de la sede con las filiales y la integración en el contexto del país anfitrión representan otra influencia en el control de la gestión.

\section{Bibliografía}

ALIBER, Robert Z. The Multinational Enterprise in a Multiple Currency World. In: DUNNING John H. (ed.). The multinational enterprise, Londres: Allen \& Unwin, 1971 $<$ https://www.abebooks.co.uk/servlet/BookDetailsPL?bi=30767240935\&searchurl=an\%3Ddu nning\%26sortby\%3D20\%26tn\%3Dmultinational\%2Benterprises\&cm_sp=snippet-_-srp1-_->.

AMBOS, Tina C. Reverse knowledge transfer. In: AUGIER, Mie y TEECE, David. (eds). The Palgrave Encyclopedia of Strategic Management. Londres: Palgrave Macmillan, 2018, p. 1.475-1476 <https://doi.org/10.1057/978-1-349-94848-2_325-1>.

AMBOS, T. C. Location choice, man-agement and performance of international R\&D investments in peripheral economies. International Journal of Technology Management, 49, 2009, p. 24-41<https://www.inderscienceonline.com/doi/abs/10.1504/IJTM.2009.024598>.

AMBOS, T. C. et al. Learning from foreign subsidiaries: An empiricalinvestigation of headquarters'benefits from reverseknowledge transfers. International Business Review, 15, 2006,

p.

294-312 <https://www.academia.edu/17483074/Learning_from_foreign_subsidiaries_An_empirical_in vestigation_of_headquarters_benefits_from_reverse_knowledge_transfers >.

ASMUSSEN, C. G. et al. Knowledge transfer and accommodation effects in mul-tinational corporations: Evidence from European sub-sidiaries. Journal of Management, 39, 2013, p. 1.397-1.429 <https://journals.sagepub.com/doi/pdf/10.1177/0149206311424316>.

ANDONOVA, Veneta; LOSADA-OTÁLORA, Mauricio. Multilatinas. Cambridge: $\begin{array}{lll}\text { Cambridge } & \text { University } & \text { Press, }\end{array}$ $<$ https://books.google.es/books?id=YhA6DwAAQBAJ\&pg=PA30\&dq=multilatinas,+definiti

\footnotetext{
${ }^{45}$ Kostova et al. 2016.

${ }^{46}$ Hopper, Tsamenyi y Wickramasinghe 2009, p. 469-514.

${ }^{47}$ Por ejemplo, Endenich et al. 2011; Kuttner y Feldbauer-Durstmueller 2016.
} 
on\&hl=es\&sa=X\&ved=2ahUKEwilwpPyhK_tAhUMahQKHTmfCM4Q6AEwAXoECAAQ Ag\#v=onepage \&q=multilatinas $\% 20 \& \mathrm{f}=$ false $>$.

ALTENBURG, Tilman. Linkages and Spillovers between Transnational Corporations and Small and Medium-Sized Enterprises in Developing Countries - Opportunities and Policies, in UNCTAD (ed.) MNC-SME Linkages for Development. Issues - experiences - best practices. UNCTAD- United Nations: Bangkok-Ginebra, 2000 <https://www.files.ethz.ch/isn/28039/2000-05.pdf>.

BAIN, Joe S. Barriers to New Competition. Cambridge: Harvard University Press, 1956 <https://doi.org/10.4159/harvard.9780674188037>.

BARNER-RASMUSSEN, Wilhelm y AARNIO, Christoffer. Shifting the faultines of language: A quantitative functional-level exploration of language use in MNC subsidiaries. Journal of World Business, 46, 2011, p. 288-295 $<$ https://reader.elsevier.com/reader/sd/pii/S1090951610000453?token=1FF446B97B6B01C04 EA492D5ECE980DF7AFE76CFB20115E5AB537DDB2433F8CFC5E61728658A0F1A63B 58FB863AE4AA9>.

BARTLETT, Christopher A. y GHOSHAL, Sumantra. Managing across borders: The transnational solution. Boston, MA: Harvard Business School Press, 1989 $<$ https://books.google.es/books?id=KYjHMVuNOAwC\&printsec=frontcover\&dq=Managing +across+borders:+The+transnational+solution.+Boston,+MA:+Harvard+Business+School+Pr ess, $+1989 . \& \mathrm{hl}=$ es\&sa=X\&ved=2ahUKEwjopezYkq_tAhV8UBUIHZWWCjEQ6AEwAHoE CAIQAg\#v=onepage \&q=Managing $\% 20$ across $\% 20$ borders $\% 3 \mathrm{~A} \% 20$ The $\% 20$ transnational $\% 2$ 0solution.\%20Boston\%2C\%20MA\%3A\%20Harvard\%20Business \%20School\%20Press \%2C $\% 201989 . \& \mathrm{f}=$ false $>$.

BELDERBOS, René et al. Intra-firm technology transfer and R\&D in foreign affiliates: Substitutes or complements? Evidence from Japanese multinational firms, Journal of the Japanese and International Economies, 22, 3, septiembre 2008, p. 310-319 $<10.1016 /$ j.jjie.2008.01.001>.

BIRKINSHAW, Julian. Strategy and management in MNE subsidiaries. In RUGMAN, Alan M. y BREWER, Thomas L. (ed.), The Oxford handbook of international business (2nd ed.), Oxford, UK: Oxford University Press, 2009, p. 367-388 $<$ https://books.google.es/books?id=gZNX61GjyhAC\&printsec $=$ frontcover\&dq=Strategy + and +management+in+MNE+subsidiaries.+In+RUGMAN,+A.M.++(Ed.),+The+Oxford+handboo $\mathrm{k}+\mathrm{of}+$ international+business \&hl=es\&sa=X\&ved=2ahUKEwit5_31kq_tAhW5TxUIHc3MAH 0Q6AEwAHoECAMQAg\#v=onepage $\& \mathrm{q}=$ Strategy $\% 20$ and $\% 20$ management $\% 20 \mathrm{in} \% 20 \mathrm{MNE}$ $\% 20$ subsidiaries.\%20In\%20RUGMAN\%2C\%20A.M.\%20\%20(Ed.)\%2C\%20The\%200xford $\% 20$ handbook\%20of\%20international\%20business\&f=false $>$.

BIRKINSHAW, Julian et al. (ed.). The Future of the Multinational Company. Chichester: $\begin{array}{llll}\text { John Wiley \& } & \text { Sons, }\end{array}$ $<$ https://books.google.es/books?id=vG1JS8myJc0C\&printsec=frontcover\&hl=es\&source=gbs _ge_summary_r\&cad $=0 \# \mathrm{v}=$ onepage \&q\&f=false>. 
BIRKINSHAW, Julian y HOOD, Neil. Multinational subsidiary evolution: Capability and charter change in foreign-owned subsidiary companies. Academy of Management Review, 23, 1998, p. 773-796 <https://doi.org/10.5465/amr.1998.1255638>.

BONAGLIA, Federico et al. Accelerated internationalization by emerging markets' multinationals: the case of the white goods sector. Journal of World Business, 42 (4), 2007, p. 369-383 <10.1016/j.jwb.2007.06.001>.

BORINI, Felipe M. et al. The reverse transfer of innovation of foreign subsidiaries of Brazilian multinationals. European Management Journal, 30 (3), 2012, p. 219-231 <https://doi.org/10.1016/j.emj.2012.03.012>.

BOSTON CONSULTING GROUP. Global Challengers: Companies on the Move: Rising Stars from Rapidly Developing Economies Are Reshaping Global Industries, Boston MA: Boston, $<$ https://www.bcgperspectives.com/content/articles/globalization_companies_on_the_move_2 011_global_challengers/>.

BUCKLEY, Peter J. y CASSON, Mark C. The Future of the Multinational Enterprise, Londres: Homes and Meier Press, 1976 <https://link.springer.com/book/10.1007/978-1-34921204-0>.

CALVO, Ángel. Estado, empresa, mercado. Standard Eléctrica y la industria de equipo de telecomunicación en España, 1926-1952, Investigaciones de Historia Económica - Economic History Research (IHE-EHR), número 13, invierno, 2009, p. 43-72 <http://diposit.ub.edu/dspace/bitstream/2445/151477/1/566324.pdf>.

CALVO, Ángel. The Emergence of Global Companies in the High-Tech Industry of Defense: The Case of Indra in Spain, 1993-2007, Eurasian Journal of Social Sciences, Eurasian Publications, vol. 7(2), 2019, p. 29-47 <https://ideas.repec.org/a/ejn/ejssjr/v7y2019i2p2947.html>.

CALVO, Ángel. World telecommunications equipment industry from the National champions to outsourcing: the case of ALCATEL, The International Journal of Business Management and Technology, Volume 3, Issue 6, November-December 2019, p. 130-152 <http://www.theijbmt.com/archive/0930/557514347.pdf>.

CALVO, Ángel. Consolidation and rationalization of the public companies in Spain: the information and communication technologies (ICT) holding, Journal of Evolutionary Studies in Business, 2019, vol. 4, núm. 1, p. 142-179 <http://hdl.handle.net/2445/127091>.

CALVO, Ángel. La reconversión en la industria de equipos de telecomunicación entre finales de la década de 1980 e inicios del siglo XXI. El caso de Alcatel-SESA, Investigaciones de Historia Económica, 16(2), 2020, p. 81-94 < https://doi.org/10.33231/j.ihe.2020.01.005>.

CALVO, Ángel. Local R\&D Capacity Building Strategies in the Two Decades Prior to the Great Recession of 2008: The Case of ICTs in Spain, European Journal of Business and Management Research, Volume-5, Issue-2, 2020a, p. 1-13 <http://www.ejbmr.org/index.php/ejbmr/article/view/274>. 
CALVO, Ángel. Technological dependence, captive market and outsourcing in the Spanish telecommunications equipment industry, International Journal of Social Research, 4:46, 2020b, p. $1-25<10.28933 /$ ijsr-2020-02-0805>.

CALVO, Ángel. Public and private investment in the roots of the mobile phone industry in Spain: Indelec, 1984-2003, International Journal of Business \& Management Studies, Volume 01; Issue 03, September 30, 2020c, p. 1-26<https://ijbms.net/current>.

CALVO, Ángel. Survival Strategies in the Spanish ICT Sector: Amper between Two Crises. International Journal of Emerging Trends in Social Sciences, 9(1), 11 de septiembre de 2020d, p. 1-20 <https://doi.org/https://doi.org/10.20448/2001.91.1.20>.

CALVO, Ángel. De empresa familiar a multinacional de las TIC: Amper 1976-2003. DTAEHE No 2009, p. 1.23 <https://www.aehe.es/wp-content/uploads/2020/09/DT-AEHE2009.pdf>.

CALVO, Ángel. La telefonía móvil celular en España: políticas de gobierno y de empresas. El caso de Indelec, 1984-2003. Historia Contemporánea, 62, 2020e, p. 219-262 <https://doi.org/10.1387/hc.20741>.

CAVES, Richard E. Multinational Enterprise and Economic Analysis, Third Edition, Cambridge: Cambridge University Press, 2007 <https://books.google.es/books?id=1_eNSlA2 WIC\&printsec $=$ frontcover\&dq=CAVES, + R.E.+Multinational+Enterprise+and+Economic + Analysis \&hl=es\&sa=X\&ved=2ahUKEwjA5NDX46ztAhWOQUEAHV3CCYkQ6AEwAHoE CAEQAg\#v=onepage \&q=CAVES\%2C\%20R.E.\%20Multinational $\% 20$ Enterprise $\% 20$ and $\% 2$ 0 Economic $\% 20$ Analysis $\& \mathrm{f}=$ false $>$.

CHANDLER, Alfred. Strategy and structure - Chapters in the history of the industrial Enterprise, $\quad$ Cambridge $\quad$ MA: $\quad$ MIT 1962. $<$ https://books.google.es/books?id=mKfjhPZTkB8C\&printsec=frontcover\&dq=CHANDLER, +Alfred,+Strategy+and+structure+\%E2\%80\%93+Chapters+in+the+history+of+the+industrial + Enterprise,+ Cambridge+MA\&hl=es\&sa $=X \& v e d=2$ ahUKEwjFqsGL5KztAhVSoVwKHSI2 CAQQ6AEwAHoECAAQAg\#v=onepage\&q\&f=false >

CHILD, John; FAILKNER, David y PITKETHLY, Robert. The Management of International Acquisitions, Londres: Oxford University 2000 $<$ https://books.google.es/books?id=dABqe8cRVqcC\&printsec=frontcover\&dq $=\mathrm{CHILD},+\mathrm{J} .+\mathrm{F}$ AULKNER,+D.+y+PITKETHLY,+ARE.+The+Management+of+International+Acquisitions $\& \mathrm{hl}=\mathrm{es} \& \mathrm{sa}=\mathrm{X} \& \mathrm{ved}=2 \mathrm{ahUKEwiFqYW} 5 \mathrm{KztAhVUTcAKHZrLD1EQ6AEwAHoECAUQAg}$ \#v=onepage \&q=CHILD\%2C\%20J.\%20FAULKNER\%2C\%20D.\%20y\%20PITKETHLY\%2 C\%20ARE.\%20The\%20Management\%20of\%20International\%20Acquisitions\&f=false>.

CRISCUOLO, Paola y NARULA, Rajneesh. Using multi-hub structures for international R\&D: Organisational inertia and the challenges of implementation, Management $\begin{array}{llllll}\text { International } & \text { Review, } & 47 & \text { (5) } & \text { 2007, } & \text { p. }\end{array}$ $<$ https://www.researchgate.net/publication/227290353_Using_multi-

hub_structures_for_international_RD_Organisational_inertia_and_the_challenges_of_implem entation>. 
DOZ, Yves L., SANTOS, José y WILLIAMSON, Peter. From global to metanational: How companies win in the knowledge economy. Cambridge MA: Harvard Business School Press, $2001<$ https://books.google.es/books?id=qzRq5KfIlwAC\&pg=PA243\&dq=DOZ,+Yves+L.,+ SANTOS,+Jos\%C3\%A9+y+WILLIAMSON,+Peter.+From+global+to+metanational:+How+ companies+win+in+the+knowledge+economy.+Harvard+Business+School+Press,+Cambridg $\mathrm{e} \& \mathrm{hl}=\mathrm{es} \& \mathrm{sa}=\mathrm{X} \& \mathrm{ved}=2 \mathrm{ahUKEwidirTLka \_ tAhWWaRUIHSLzD2cQ6AEwAHoECAIQAg \# v}$ $=$ onepage $\& q=\mathrm{DOZ} \% 2 \mathrm{C} \% 20 \mathrm{Yves} \% 20 \mathrm{~L} . \% 2 \mathrm{C} \% 20 \mathrm{SANTOS} \% 2 \mathrm{C} \% 20 \mathrm{~J}$ os $\% \mathrm{C} 3 \% \mathrm{~A} 9 \% 20 \mathrm{y} \% 20$ WILLIAMSON\%2C\%20Peter.\%20From\%20global\%20to\%20metanational\%3A\%20How\%2 0companies $\% 20$ win $\% 20$ in $\% 20$ the $\% 20$ knowledge $\% 20$ economy. $\% 20 \mathrm{Harvard} \% 20 \mathrm{Business} \% 2$ 0School\%20Press \%2C\%20Cambridge \&f=false $>$.

DUNNING, John H. The Eclectic (OLI) Paradigm of International Production: Past, Present and Future. Journal of Economics of Business, 8(2), 2001, p. 173-190 <https://www.tandfonline.com/doi/abs/10.1080/13571510110051441>.

DUNNING, John H. The Eclectic Paradigm of International Production: A Restatement and Some Possible Extensions. Journal of International Business Studies, 19 (1), (1988), p. 1-31 <https://www.jstor.org/stable/154984?seq=1>.

DUNNING, John H. International Production and the Multinational Enterprise. Londres: George Allen and Unwin, $1981<$ https://www.routledge.com/International-Production-andthe-Multinational-Enterprise-RLE-International/Dunning/p/book/9781138007840>.

DZEDEK, Lars R. Initiatives by Subsidiaries of Multinational Corporations: An Empirical Study on the Influence of Subsidiary Role Context. Fort Lauderdale: Springer, 2018 $<$ https://books.google.es/books?id=1q9JDwAAQBAJ\&printsec $=$ frontcover $\&$ hl $=$ es\&source $=\mathrm{g}$ bs_ge_summary_r\&cad $=0 \# v=$ onepage $\& q \& \mathrm{f}=$ false $>$.

EDWARDS, Tony y TEMPEL, Anne. Explaining variation in reverse diffusion of HR practices: Evidence from the German and British subsidiaries of American multinationals, $\begin{array}{llllll}\text { Journal of } & \text { World } & \text { Business, } 45 & \text { (2010), } & \text { p. }\end{array}$ <https://reader.elsevier.com/reader/sd/pii/S1090951609000261 ?token=714DC65F874D63117 0DA4241B4793C01BBC2283891B856A3CA8BCB59474D9E10938F98D89E52D827E8352 94582559E59>.

GHOSHAL, Sumantra; BARTLETT, Christopher A. Creation, Adoption and Diffusion of Innovations by Subsidiaries of Multinational Corporations. Journal of International Business Studies, volumen 19,1988a, p. 365-388 <https://econpapers.repec.org/article/paljintbs/v_3a19_3ay_3a1988_3ai_3a3_3ap_3a365388.htm>.

EGELHOFF, William G. Patterns of Control in US, UK, and European Multinational Corporations. Journal of International Business Studies, 15, 1984, p. 73-83 $<$ https://www.jstor.org/stable/154233?seq=1>.

ENDENICH, Christoph, BRANDAU, Michael; HOFFJAN, Andreas. Two decades of research on comparative management accounting - achievements and future directions, Austin Accounting Review, 21, 2011, p. 365-382 <https://www.kau.edu.sa/Files/0003203/Subjects/Two\%20Decades\%20of\%20Research\%20o n\%20Comparative\%20Management.pdf $>$. 
FARLEY, John U.; HOENIG, Scott; YANG, John Z. Key factors influencing HRM practices of overseas subsidiaries in China's transition economy, International Journal of Human Resource Management, 15 (5), agosto, 2004, p. 688-704 <https://www.academia.edu/7233383/Key_factors_influencing_HRM_practices_of_overseas _subsidiaries_in_Chinas_transition_economy>.

FOSFURI, Andrea; MOTTA, Massimo. Multinationals without advantages. Scandinavian Journal of Economics, 1999, p. 617-630 <https://doi.org/10.1111/1467-9442.00176>.

FREITAS, Franciane et al. Determinants of reverse knowledge transfer for emerging market multinationals: the role of complexity, autonomy and embeddedness. Revista de Administração, volumen 52, 2, abril-junio de 2017, p. 176-188 <http://dx.doi.org/10.1016/j.rausp.2016.12.007>.

FROST, Tony S. The geographic sources of innovation in the multinational enterprise: US subsidiaries and host country spillovers, 1980-1990. Boston: Massachusetts Institute of Technology, $1998<$ https://dspace.mit.edu/handle/1721.1/9687>.

FROST, Tony S. ; ZHOU, Changhui. R\&D co-practice and 'reverse' knowledge integration in multinational firms. Journal of International Business Studies, volumen 36, 2005, p. 676687 <http://www.rcmewhu.com/upload/file/20150528/20150528151907_9205.pdf>.

GAMBLE, Jos. Transferring human resource practices from the United Kingdom to China: the limits and potential for convergence. International Journal of Human Resource $\begin{array}{llllll}\text { Management. } & 14 & \text { (3), 2003, } & \text { p. } & 369-387\end{array}$ $<$ https://www.researchgate.net/publication/247522458_Transferring_human_resource_practic es_from_the_United_Kingdom_to_China_The_limits_and_potential_for_convergence>.

GHOSHAL, Sumantra y BARTLETT, Christopher A. The multinational corporation as an interorganizational network, Academy of Management Review. 15 (4), 1990, p. 603-625 <https://pdfs.semanticscholar.org/2082/8ab1ee7ed146a64b82c756f5d04d8fbd8ee9.pdf>.

GHOSHAL, Sumantra; BARTLETT, Christopher A. Creation, Adoption and Diffusion of Innovations by Subsidiaries of Multinational Corporations. Journal of International Business Studies, volumen 19, 1988a, p. 365-388 <https://flora.insead.edu/fichiersti_wp/Inseadwp1988/88-31.pdf>.

HAKANSON, Lars; NOBEL, Robert. Organizational characteristics and reverse knowledge transfer. Management International Review, 41, 2001, p. 395-420 <https://www.scielo.br/scielo.php?script=sci_arttext\&pid=S1807-76922017000100305>.

HANSEN, Morten T.; NOHRIA, Nitin. Organizing Multinational Companies for Collaborative Advantage. In QUELCH, John y DESHPANDÉ, Rohit eds. The Global Market: Developing a Strategy to Manage Across Borders. San Francisco: John Wiley \& Sons, 2004, p. 92-112 <https://epdf.pub/the-global-market-developing-a-strategy-to-manageacross-borders-wiley-desktop-e.html $>$.

HARRISON, Graeme L.; MCKINNON, Jill. Cross-cultural research in management control systems design: A review of the current state. Accounting, Organizations and Society, 24 (5- 
$6)$,

1999

p.

483-506

$<$ https://citeseerx.ist.psu.edu/viewdoc/download?doi=10.1.1.468.3347\&rep=rep1\&type=pdf $>$.

HARZING, Anne-Wil. Managing the Multinationals: An international study of control mechanisms, Cheltenham, UK y Northampton MA: Edward Elgar Publishing Limited, 1999 <https://books.google.es/books?id=TspqQgAACAAJ\&dq=HARZING,+Anne-

Wil.+Managing+the+Multinationals:+An+international+study+of+control+mechanisms\&hl= es\&sa=X\&ved=2ahUKEwiOofiz8qztAhUN4OAKHY_oAvUQ6AEwAHoECAAQAg>.

HARZING, Anne-Wil; KÖSTER, Kathrin y MAGNER, Ulrike. Babel in business: The language barrier and its solutions in the HQ-subsidiary relationship. Journal of World $\begin{array}{llll}\text { Business, } & 46 & \text { (2011), } & \text { p. }\end{array}$ <https://reader.elsevier.com/reader/sd/pii/S1090951610000441?token=60807F6A194C1A410 9CBE4F9205F1B63AC602338F39E8A199E34E7A60209776DA286D7620FF99294667B25 C8CB895C8A>.

HEIKKILÄ, Jukka-Pekka; SMALE, Adam. The effects of 'language standardization' on the acceptance and use of e-HRM systems in foreign subsidiaries. Journal of World Business, 46, 2011, p. 305-313 <https://reader.elsevier.com/reader/sd/pii/S109095161000043X?token=7181DCBCA8446936 F440FC06838F5FC1ADEE431CF25F65922189092FEE0988F29C90B01D2CC2B843F01E1 7D43EEE7F73>.

HILL, Charles W. L. et al. Strategic Management: Theory: An Integrated Approach. Cengage Learning, Samford CT, 2014 $<$ https://books.google.es/books?id=_MKiAgAAQBAJ\&pg=PR4\&dq=Strategic+Management :+Theory:+An+Integrated+Approach.+Cengage+Learning,+Samford+CT,+2014.\&hl=es\&sa= $\mathrm{X} \& v e d=2$ ahUKEwislITJrOztAhWm3eAKHayIACYQ6AEwAHoECAIQAg\#v=onepage \&q= Strategic\%20Management\%3A\%20Theory\%3A\%20An\%20Integrated\%20Approach.\%20Ce ngage $\% 20$ Learning $\% 2 \mathrm{C} \% 20$ Samford $\% 20 \mathrm{CT} \% 2 \mathrm{C} \% 202014 . \& \mathrm{f}=$ false $>$.

HOLM, Ulf; PEDERSEN, Torben. The emergence and impact of MNC centres of excellence: A subsidiary perspective. Londres: Macmillan, 2000 $<$ https://d1wqtxts1xzle7.cloudfront.net/8296228/2001-03.pdf?1328343388=\&responsecontentdisposition=inline\%3B+filename\%3DThe_emergence_and_impact_of_MNC_centres.pdf\&E xpires $=1606992154 \&$ Signature=DRG2w1ur9QZkh0JoBEvjTKGMZLzg6ram6a2t8p3cU588VCYeAUChUY-

BeI2V5MNaSc9N1vgLowVqEg5ANtVnHj5klid9Nh8JMrHwFyW8XJAiSm92KDFvZRGWTHdlNoTR0U7CHmXqP9MbU116tPnUXmsRB07xqCiLp2v61RhYGJzsW0 X5BHXutB0cwbumrqeDgvMnzB6H QiCEHrU0Q->.

HOPPER, Trevor; TSAMENYI, Mathew; WICKRAMASINGHE, Danture. U. S. Management accounting in less developed countries: what is known and needs knowing. Account Audit Account Journal, 22, 2009, p. 469-514 <https://www.emerald.com/insight/content/doi/10.1108/09513570910945697/full/html>.

HYMER, Stephen H. The International Operations of National Firms: A Study of Foreign Direct Investment, PhD Dissertation, Cambridge: MIT Press, 1976 [1960] $<$ https://dspace.mit.edu/handle/1721.1/27375>. 
JAUSSAUD, Jacques; SCHAAPER, Johannes. European and Japanese Multinational Companies in China: Organization and Control of Subsidiaries, Asian Business \& Management, 6(3), septiembre 2007, p. 223-245 <10.1057/palgrave.abm.9200222>.

JIMENEZ, Daniel; MARTÍNEZ-COSTA, Micaela; SANZ, Raquel. Reverse knowledge transfer and innovation in MNCs. European Journal of Innovation Management, July 2019, p. 629-648 <https://doi.org/10.1108/EJIM-10-2018-0226>.

JOHNSON, Karen L.; DUXBURY, Linda. The view from the field: A case study of the expatriate boundary-spanning role. Journal of World Business, 45 (2010), p. 29-40 $<$ https://reader.elsevier.com/reader/sd/pii/S1090951609000273?token=3F87B20CEC1E34646 E3A94BAD6AD353DE4E3666180184C84D44881A2C4271DD013A62F62F15F851C862A 30A3BA6026E2>.

KHAN, Rahat N. Multinational companies and the world economy: economic and technological impact, Impact of science on society, 141, 1986, p. 15-25 $<$ https://scholar.google.es/scholar?cluster $=10679689246982464071 \& \mathrm{hl}=$ es\&as_sdt=2005\&sc iodt $=0,5>$.

KIM, Chanv W.; HWANG, Peter. Global Strategy and Multinationals' Entry Mode Choice. Journal of International Business Studies, volumen 23, 1992, pp 29-53 <https://doi.org/10.1057/palgrave.jibs.8490258>.

KLITMØLLER, Anders; LAURING, Jakob. When global virtual teams share knowledge: Media richness, cultural difference and language commonality. Journal of World Business, 48 (2013), p. 398-406 <https://reader.elsevier.com/reader/sd/pii/S1090951612000764?token=AC9437F082E49047C 2C56FB12AE3BA14BD8796D5C8162B280635E5223F375C574B218AA1C86031763DEC5 4094E4DE2B2>.

KOGUT, Bruce; ZANDER, Udo. Knowledge of the Firm and the Evolutionary Theory of the Multinational Corporation. Journal of International Business Studies, 34(6), 2003, p. 516-530 <file:///C:/Users/Angel/AppData/Local/Temp/Kogut-

Zander1993_Article_KnowledgeOfTheFirmAndTheEvolut.pdf>.

KOGUT, Clarice; COTTA DE MELLO, Renato. Reverse Knowledge Transfer in Multinational Companies: A Systematic Literature Review, Administration Review 14(1), enero de 2017, p. 1590-1807 <http://www.anpad.org.br/bar>.

KOSTOVA, Tatiana et al. Headquarters-subsidiary relationships in MNCs: fifty years of evolving research. Journal of World Business, 51, 2016, p. 176-184 <https://www.sciencedirect.com/science/article/pii/S1090951615000796\#sec0010>.

KUTTNER, Michael; FELDBAUER-DURSTMUELLER, Birgit. Comparative management accounting in developing countries-state-of-the-art and future perspectives. International Journal of Business Research, 16, 2016, p. 81-104 <https://www.researchgate.net/publication/304169921_Comparative_management_accountin g_in_developing_countries_-_State-of-the-art_and_future_perspectives $>$. 
LUNNAN, Randi et al. Dealing with headquarters in the multinational corporation: a subsidiary perspective on organizing costs. Journal of Organization Design, 8, 12, 2019, p. 1$24<$ https://doi.org/10.1186/s41469-019-0052-y>.

MACDOUGALL, G. D. A. The Benefits and Costs of Private Investments from Abroad: A Theoretical Approach. Economic Record, 36, 1960, p. 15-35 <https://doi.org/10.1111/j.14680084.1960.mp22003002.x>.

MARIN, Daila; ROUSOVÁ, Linda Fache. The Organization of European Multinationals, $\begin{array}{llll}\text { CEPR Discussion } & \text { Papers, } & 8.881, & 2011\end{array}$ $<$ https://papers.ssrn.com/sol3/papers.cfm?abstract_id=2034099>.

MARKUSEN, James R.; VENABLES, Anthony J. Foreign Direct Investment as a Catalyst for Industrial Development. European Economic Review, 43, 1999, p. 335-356 <http://www.sciencedirect.com/science/article/pii/S0014-2921(98)00048-8>.

MATHEWS, John A. Dragon multinationals: New players in $21^{\text {st }}$ century globalization, Asia Pacific Journal of Management, volumen 23, 2006, p. 5-27 $<$ https://link.springer.com/article/10.1007\%2Fs10490-006-6113-0>.

NAIR, Smitha R.; MELLAHI, Kamel. Reverse Knowledge Transfer from Overseas Acquisitions: A Survey of Indian MNEs. Management International Review, volumen 55, 2015, p. 277-301<https://doi.org/10.1007/s11575-015-0242-y>, $<$ https://link.springer.com/article/10.1007/s11575-015-0242-y>.

O'DONNELL, Sharon. Managing Foreign Subsidiaries: Agents of Headquarters, or an Interdependent Network?, Strategic Management Journal, 21, 2000, p. 525-548 $<10.1002 / \% 28$ SICI\%291097-0266\%28200005\%2921\%3A5\%3C525\%3A\%3AAIDSMJ104\%3E3.0.CO\%3B2-Q>.

O'DONNELL, Sharon; BLUMENTRITT, Timothy. The Contribution of Foreign Subsidiaries to Host Country National Competitiveness. Journal of International Management, 5, 1999, p. 187-206 <https://doi.org/10.1016/S1075-4253(99)00012-5>.

PAIK, Yongsun; SOHN, Junghoon Derick. Expatriate managers and MNC's ability to control international subsidiaries: the case of Japanese MNCs. Journal of World Business, Volume 39, $1, \quad$ febrero $\quad 2004, \quad$ p. $\quad 61-71$ <https://www.sciencedirect.com/science/article/pii/S1090951603000361>.

ROSENZWEIG, Philip M. The new "American challenge": Foreign multinationals in the United States. California Management Review, 36 (3), (1994), p. 107-123 <https://journals.sagepub.com/doi/abs/10.2307/41165757>.

PAPANASTASSIOU, Marina; PEARCE, Robert. The Strategic Development of Multinationals: Subsidiaries and Innovation. Londres: Springer, 2009 $<$ https://books.google.es/books?id=E5rWCwAAQBAJ\&printsec=frontcover \&hl=es\&source= gbs_ge_summary_r\&cad $=0 \# \mathrm{v}=$ onepage $\& \mathrm{q} \& \mathrm{f}=$ false $>$.

PORTER, Michael E. Competitive Advantage of nations. Nueva York: The Free Press, 1990 $<$ https://books.google.es/books?id=CqZzxAxBpfEC\&pg=PR4\&dq=PORTER,+Michael+E.+ 
Competitive + Advantage.+ Nueva + York $:+$ The + Free + Press,$+1990 . \&$ hl $=$ es $\& s a=X \& v e d=2 a h U$ KEwiogdnq1bHtAhWMYsAKHTguA50Q6AEwBnoECAUQAg\#v=onepage \&q=PORTER\% 2C\%20Michael\%20E.\%20Competitive\%20Advantage.\%20Nueva\%20York\%3A\%20The\%20 Free $\% 20$ Press $\% 2 C \% 201990 . \& f=$ false $>$.

POSADA, José C.; RUIZ, Francisca. Sistemas regionales de innovación: universidad y empresa <https://old.aecr.org/web/congresos/1997/cts/comun/a2/02-219fjj〉.

PRAHALAD, C. K.; DOZ, Yves L. The multinational mission: Balancing local demands and global vision, Nueva York: $\quad$ Free $\quad$ Press, 1987 $<$ https://books.google.es/books?id=Pc1zfvMqNAoC\&printsec $=$ frontcover\&dq $=$ The + multinati onal+mission:+Balancing+local+demands+and+global+vision $\& h l=e s \& s a=X \& v e d=2 a h U K E$ wiBzpWQt-

btAhUIQkEAHRJRCewQ6AEwAHoECAAQAg\#v=onepage \&q=The $\% 20$ multinational $\% 20$ mission $\% 3 \mathrm{~A} \% 20 \mathrm{Balancing} \% 20$ local\%20demands\%20and $\% 20$ global\%20vision $\& \mathrm{f}=$ false $>$.

RAMAMURTI, Ravi; SINGH, Jitendra. Emerging multinationals in emerging markets. Cambridge: Cambridge University $\quad$ Press, 2009 $<$ https://books.google.es/books?id=NHYBTh4qrSoC\&printsec=frontcover\&hl=es\&source=g bs_ge_summary_r\&cad=0\#v=onepage $\& q \& \mathrm{f}=$ false 2009>.

REICHE, B. Sebastian. Knowledge transfer in multinationals: The role of inpatriates' boundary spanning. Human Resource Management, Volume 50, Issue 3, mayo/junio de 2011, p. 365-389<https://doi.org/10.1002/hrm.20423>.

RODRIGUEZ-CLARE, Andrés, Multinationals, linkages, and economic development, The American Economic Review, 86, No. 4, septiembre, 1996, p. 852-873 $<\mathrm{http} / / / \mathrm{www}$.jstor.org/stable/211>.

ROSADO, Ana. Barriers to Competition: The Evolution of the Debate. Routledge: Abingdon OX,

$<$ https://books.google.es/books?id=IVykCgAAQBAJ\&printsec $=$ frontcover\&hl=es\&source $=\mathrm{g}$ bs_ge_summary_r\&cad $=0 \# v=$ onepage $\& q \& f=$ false $>$.

RUGMAN, Alan M. The Regional Multinationals: MNEs and 'Global' Strategic Management. Cambridge: Cambridge University Press, 2005 $<$ https://books.google.es/books?id=BgDnv2XlssQC\&printsec=frontcover\&hl=es\&source=gbs _ge_summary_r\&cad $=0 \# \mathrm{v}=$ onepage $\& \mathrm{q} \& \mathrm{f}=$ false $>$.

RUGRAFF, Eric; HANSEN, Michael W. (eds.). Multinational Corporations and Local Firms in Emerging Economies. Amsterdam: Amsterdam University Press, 2011 <https://pdfs.semanticscholar.org/5a15/e5bfab7e0b05c340fecd10c2dd6444018840.pdf >.

SAGEDER, Martina; FELDBAUER-DURSTMÜLLER, Birgit. Management control in multinational companies: a systematic literature review. Review of Managerial Science, volume 13, p. 875-918 <https://link.springer.com/article/10.1007/s11846-018-0276-1>

SCHAAPER, Johannes et al. Control of French and Japanese subsidiaries in China: implementing control mechanisms before and after the global economic crisis. Asia Pacific 
Las multinacionales y las relaciones con sus filiales

Business Review, 17, 2011, p. <https://www.tandfonline.com/doi/abs/10.1080/13602381.2011.546626>.

SHETTY, Y. Krishan. Managing the Multinational Corporation: European and American Styles. Management International Review, Vol. 19, No. 3 (1979), p. 39-48 $<$ https://www.jstor.org/stable/40227469?seq=1>.

STOPFORD, John M.; STRANGE, Susan y HENLEY, John S. Rival States, Rival Firms: Competition for World Market Shares, Cambridge: Cambridge University Press, 1991 $<$ https://books.google.es/books?id=k1oVv2ZejJcC\&printsec=frontcover\&hl=es\&source=gbs_ ge_summary_r\&cad $=0 \# \mathrm{v}=$ onepage $\& \mathrm{q} \& \mathrm{f}=$ false $>$.

TEECE, David J. Technology Transfer by Multinational Firms: The Resource Cost of Transferring Technological Know-How. Economic Journal, 87, junio, 1977, p. 242-61 <https://ideas.repec.org/a/ecj/econjl/v87y1977i346p242-61.html>.

TIPPMANN, Esther; SHARKEY Scott, P.; MANGEMATIN, Vincent. Subsidiary managers' knowledge mobilizations: Unpacking emergent knowledge flows. Journal of World Business, 49, (2014), p. 431-443 <https://reader.elsevier.com/reader/sd/pii/S1090951613000606?token=248036B320FF41D99 11AFCA80BDA39E5E5AF69050F00348F3E326D9EABD38E203844074E429E33FB6D9B 781435E9E4F9>.

UNITED NATIONS. Multinational Corporations in World Development, Nueva York: United Nations, 1973 <https://digitallibrary.un.org/record/819904?ln=es>.

UNITED NATIONS, The impact of multinational corporations on development and on international relations, Nueva York: United Nations, 1974 $<$ https://digitallibrary.un.org/record/819904?ln=es>.

VACHANI, Sushil. Enhancing the Obsolescing Bargain Theory: A Longitudinal Study of Foreign Ownership of US and European Multinationals. Journal of International Business Studies, 26, 1995, 2 p. 159-80 <https://www.researchgate.net/publication/228560706_From_the_obsolescing_bargain_to_th e_political_bargaining_model>.

VERNON, Roger. International Investment and International Trade in the Product Cycle, The Quarterly Journal of Economics, vol. 80, issue 2, 1966, p. 190-207 $<$ http://bev.berkeley.edu/ipe/readings/International\%20Investment $\% 20$ and $\% 20$ International\% 20Trade\%20in\%20the\%20Product\%20Cycle.pdf $>$.

YAMAO, Sachiko; SEKIGUCHI, Tomoki. Employee commitment to corporate globalization: The role of English language proficiency and human resource practices. Journal of World $\begin{array}{llll}\text { Business, } & 50 & \text { (2015), } & \text { p. }\end{array}$ $<$ https://reader.elsevier.com/reader/sd/pii/S109095161400025X?token=09C42530244890017 417ADC777AF2F82A3870EF2C921EADAF1DCD9533C5FCCD75DC9153CC63E931B45 B962C86671109D>. 
YAMIN, Mo. Determinants of reverse transfer: The experience of UK multinationals. In New challenges for European and International Business, proceedings of the 21st annual EIBA conference. Urbino: Schiattarella, 1995 <https://www.eiba.org/r/past-conferences>.

ZANDER, Lena; MOCKAITIS, Audra Irene; BUTLER, Christina L. Leading global teams. $\begin{array}{lllll}\text { Journal of World } & \text { Business, } 47 & \text { (2012), } & \text { p. }\end{array}$ <https://isiarticles.com/bundles/Article/pre/pdf/4606.pdf $>$.

Ficha bibliográfica:

CALVO, Ángel. Las multinacionales y las relaciones con sus filiales. Ar@cne. Revista Electrónica de Recursos de Internet sobre Geografia y Ciencias Sociales. Barcelona: Universidad de Barcelona, I de mayo de 202I, vol. XXV, n² 254. DOI: https://doi.org/I0.I344/ara202I.254.34732

\author{
Menú Geo Crítica
}

